\title{
A Power Quality Monitoring System Via the Ethernet Network Based on the Embedded System
}

\author{
Krisda Yingkayun ${ }^{1}$ and Suttichai Premrudeepreechacharn ${ }^{2}$ \\ ${ }^{1}$ Rajamangala University of Technology Lanna, \\ ${ }^{2}$ Chiang Mai University \\ Thailand
}

\section{Introduction}

The problems about power quality have increasingly caused a failure or a malfunction of the end user equipment for the past few years up to now. The problems have concerned with either voltage or current frequency deviation. To have the power quality monitoring done flowingly and completely, the measurement takes an important role on voltage, current, frequency, harmonic distortion and waveforms. Many researchers have used methods of power quality measurement (Dugan et al., 2002; Baggini, 2008) while other researchers have used various protocols to control the system (Auler \& d'Amore, 2002). Others have presented the data acquisition based on PC (Batista et al., 2003) or Power Line Communication (Hong et al., 2005) or TMS320CV5416 DSP Processor (Rahim bin Abdullah \& Zuri bin Sha'ameri, 2005). Another researcher has applied ARM and DSP processor (Yang $\&$ Wen, 2006) or has only applied DSP processor (Salem et al., 2006) to monitoring power quality in real time. In the meantime, the detecting fault signals of power fluctuation in real time and a power quality monitoring for real-time fault detection using real-time operating system (RTOS) are proposed (Yingkayun \& Premrudeepreechacharn, 2008,2009) and the low cost power quality monitoring system is suggested (So et al., 2000; Auler \& d'Amore, 2009), for example.

This chapter has developed the idea of power quality monitoring system via the Ethernet network based on the embedded system with the two selected ARM7 microcontrollers: ADUC7024 and LPC2368. On account of ADUC7024, it has a function of sampling waveforms and of writing the sampling signals to the external memory. Meanwhile, LPC2368 can execute the main tasks: detecting the fault signals; storing fault data in SDCARD up to $2 \mathrm{~GB}$; and communicating with PC or laptop via the Ethernet network. The power quality monitoring on the embedded system suggested can acquire the voltage, the status and the frequency. It can send them via network at real time, can operate as stand alone equipment and can display the fault signals in real time of power fluctuation. But anyhow, when being absent, we can download the fault data from the site place, depending on the program configuration. In this case the fault signals can be displayed on the screen of the PC or laptop at real time or can be done after as desired. Moreover, there can be a single 
phase or a 3-phase voltage measurement supported by the power quality monitoring hardware. When working in different places, we can establish the network in various sites and connect via the Ethernet network from a single PC or laptop. The network has the capability to send from the board site to the PC or laptop with high speed up to 100 megabit per second $(\mathrm{Mb} / \mathrm{s})$. Nevertheless, it is easy to monitor the power quality monitoring system via the Ethernet network from PC or laptop.

This chapter is organized as follow: Section 2 is the architecture of the power quality monitoring being composed of 2 sets of ARM7 microcontroller boards, 3 signal conditioning modules, external memory board and energy measurement board. Section 3 is the embedded software design with the details of sampling concept, power quality monitoring concept, data frames, configuration data and Ethernet packet structures. Section 4 is the application software to interface the power quality monitoring hardware. Section 5 is the experimental results which are displaying the fault signals from AC lines while being on operation or being done after. Section 6 is the conclusion from the research with the future work suggestion.

\section{Architecture of the power quality monitoring system}

The architecture of the power quality monitoring system is planned for specific purposes which are to detect, to store, to download and to display the fault signals while being on operation or being done later on. The main structures are divided into 5 circuit boards. The block diagram of the architecture of the power quality monitoring system is shown in Fig. 1.

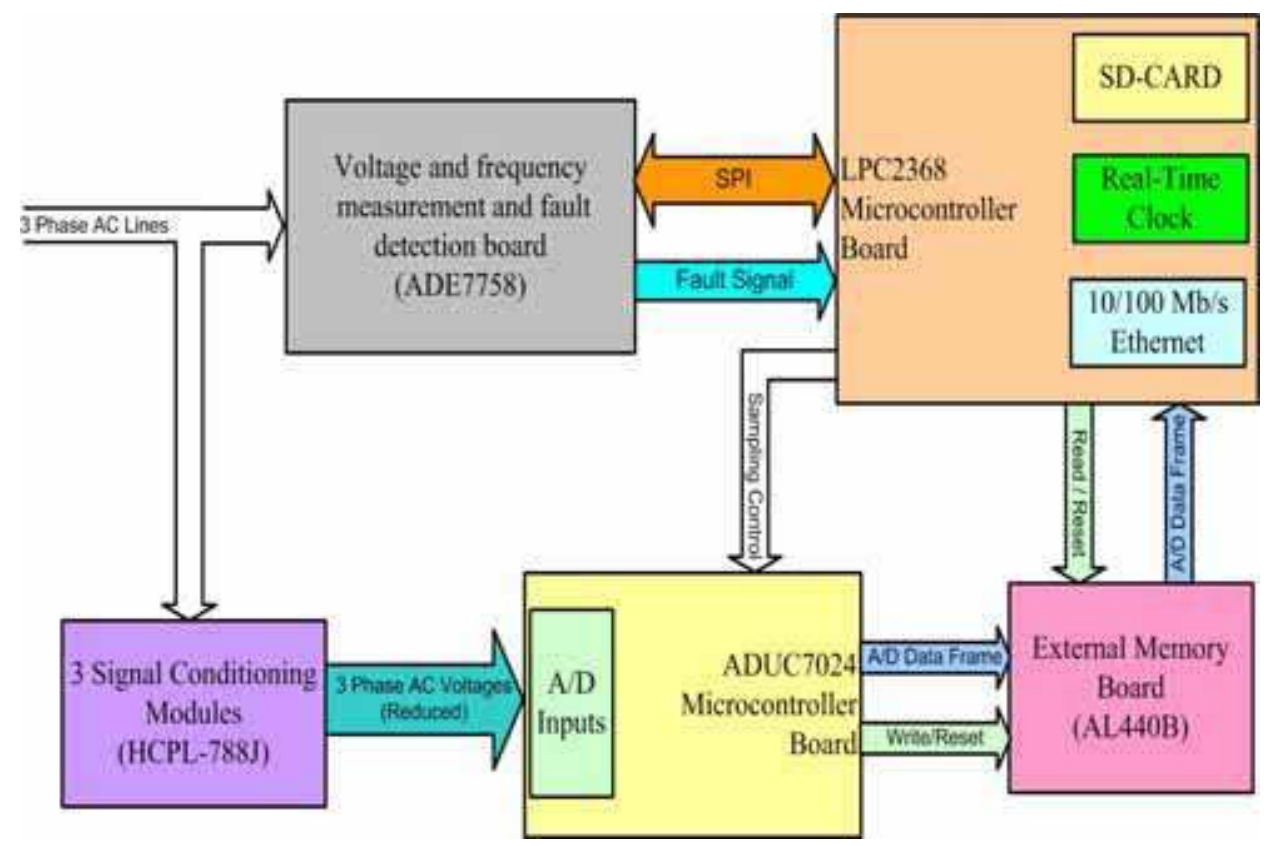

Fig. 1. Block diagram of the architecture of the power quality monitoring system 


\subsection{Signal conditioning modules (HCPL-788J)}

There are actually 3 signal conditioning modules with 3 HCPL-788J integrated circuits for measuring the voltage of the three-phase AC lines in order to send the attenuated AC voltage to the waveform sampling board which the ADUC7024 microcontroller is embedded in. The 3 signal conditioning modules have used the same electronic circuit. The signal conditioning circuit is shown in Fig. 2.

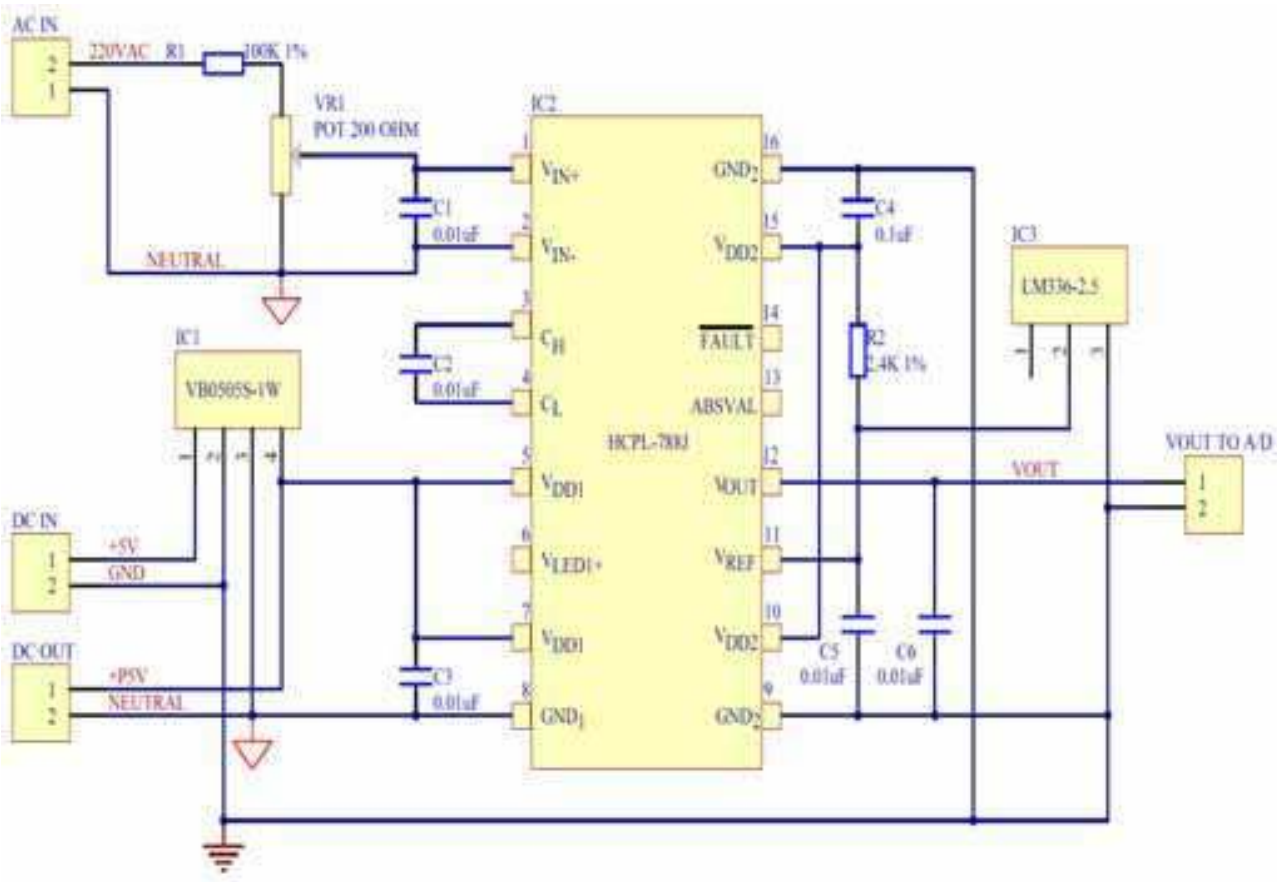

Fig. 2. The circuit of the signal conditioning module

\subsection{The voltage and frequency measurement and fault detection board (ADE7758)}

This board is the key part of monitoring system of fault detection which refers to sags and overvoltages. In this chapter, ADE7758 integrated circuit has been chosen to operate because of its suitable qualification to detect the fault signals in time. The circuit of the board is shown in Fig. 3.

\subsection{Microcontroller boards (ADUC7024 and LPC2368)}

As it is known that the embedded system with the two selected ARM7 microcontrollers which are ADUC7024 and LPC2368 are the developed microcontroller boards, using in this chapter. The first board is used for sampling waveforms and for writing the sampling signals to the external memory and the latter is used for various purposes: (1) reading voltage, frequency and fault detection from ADE7758; (2) storing fault data in SD-CARD up to $2 \mathrm{~GB}$, for communicating with PC or laptop via the Ethernet Network and; (3) controlling the sampling process of the ADUC7024 board. The picture of the two microcontroller boards are shown in Fig. 4 and Fig. 5. 


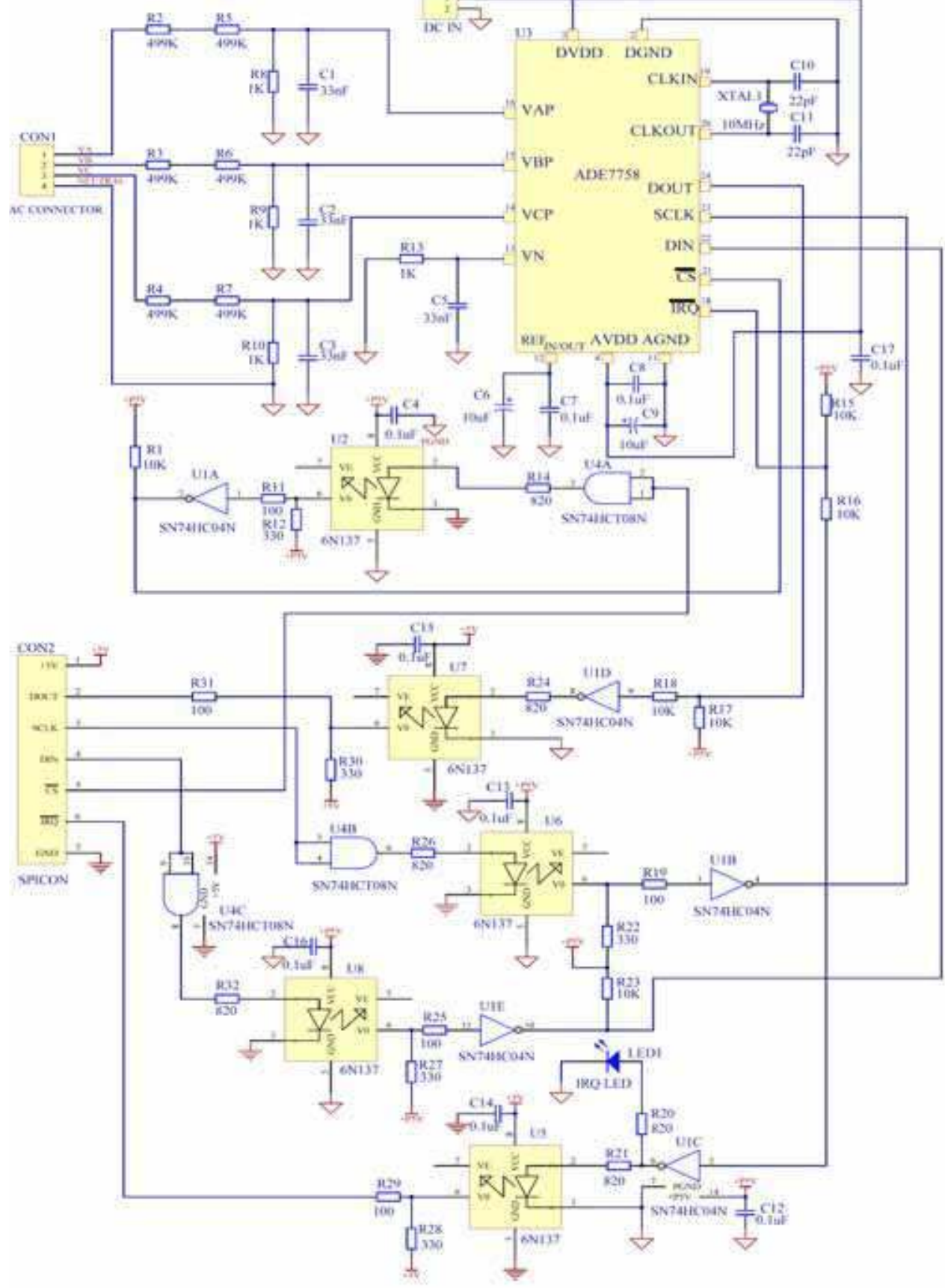

Fig. 3. The circuit of the voltage and frequency measurement and fault detection board 


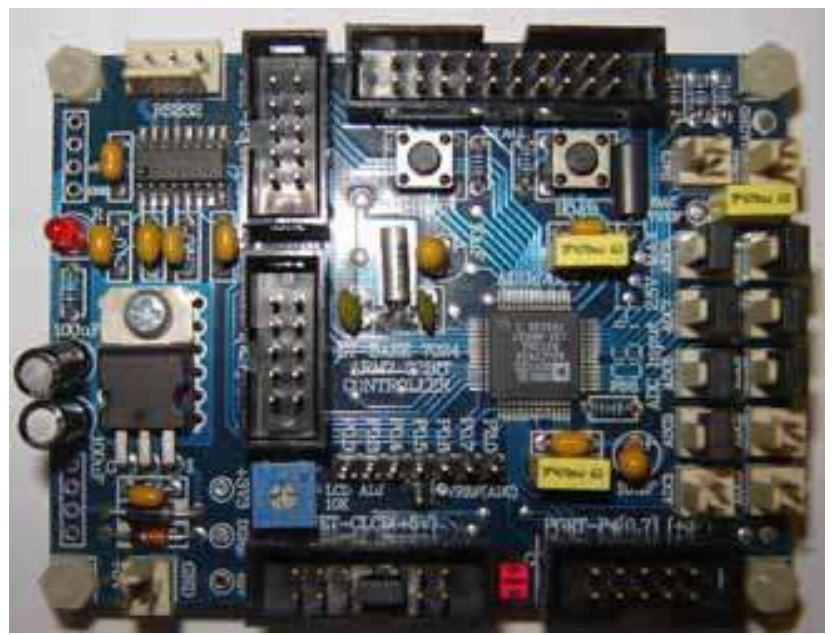

Fig. 4. The development board of ADUC7024 microcontroller

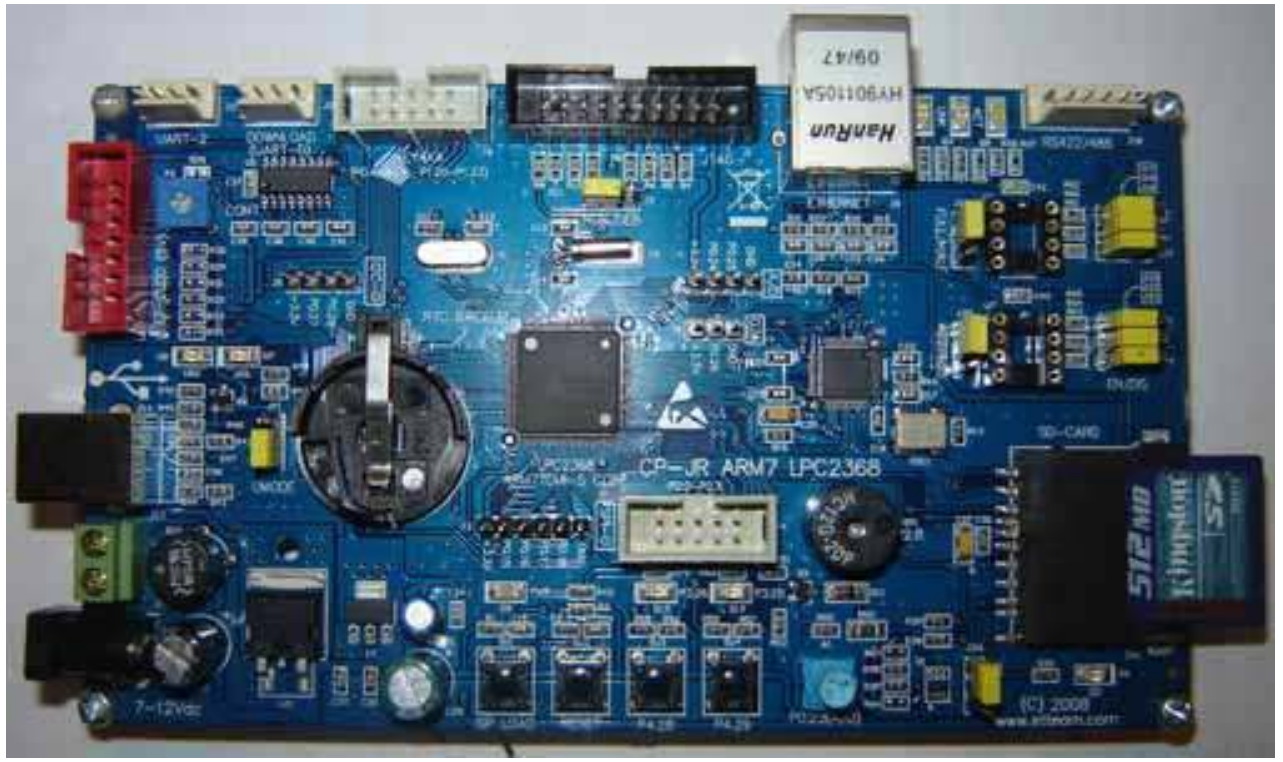

Fig. 5. The development board of LPC2368 microcontroller

\subsection{External memory board (AL440B)}

This external memory board is one device of this power quality monitoring architecture to collect the fault data from ADUC7024 microcontroller into its memory in series. The data will be read by the LPC2368 microcontroller board and will be stored in SD-CARD for recalling or downloading later via the Ethernet network when of necessity needed. The circuit of the external memory board is shown in Fig. 6. 


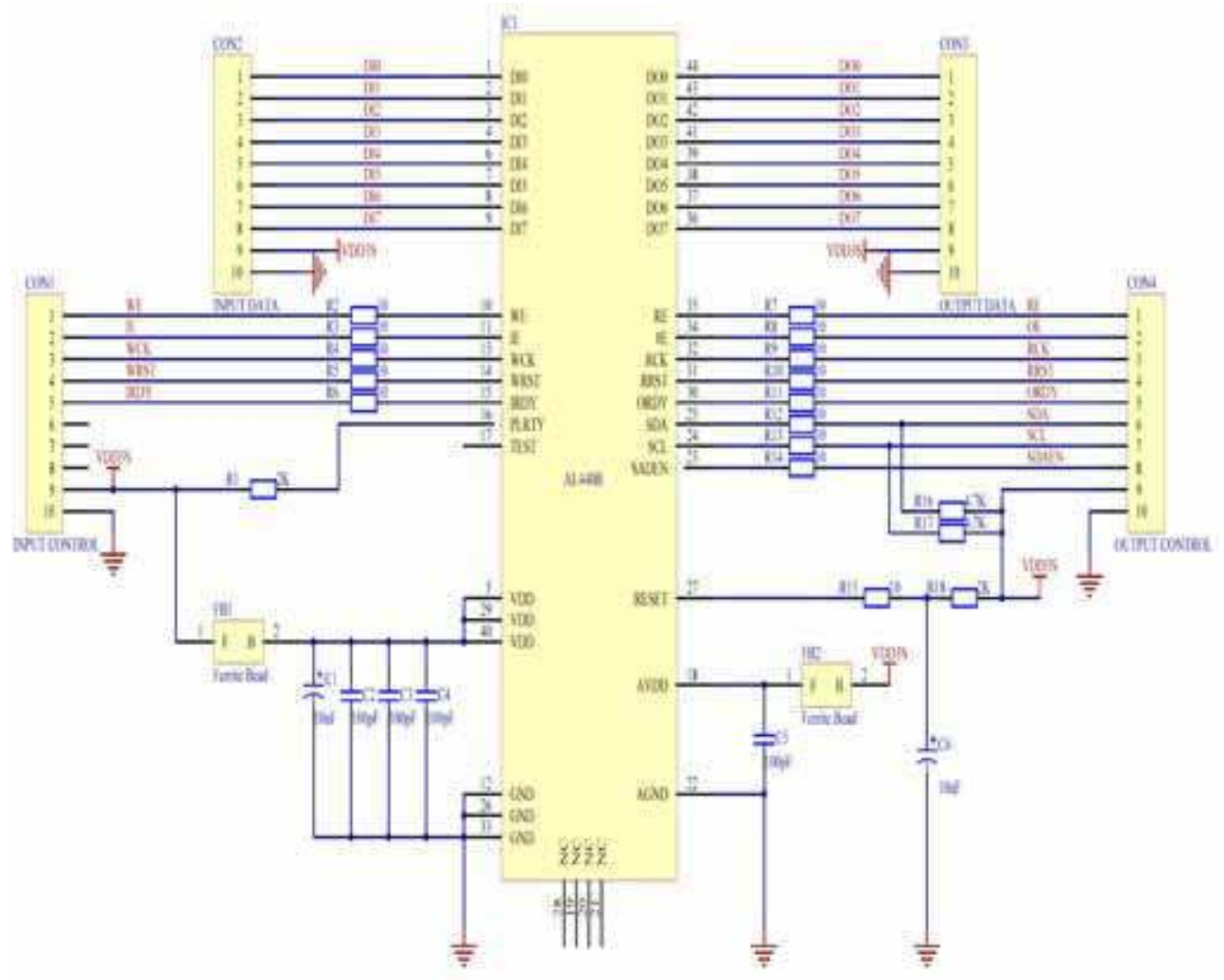

Fig. 6. The circuit of the external memory board

\subsection{The process of the power quality monitoring system}

The picture of the power quality monitoring system hardware is shown in Fig. 7, consisting of five parts as the followings:

1. The voltage and frequency measurement and fault detection board (ADE7758)

2. Signal conditioning module board (HCPL-788J)

3. ADUC7024 microcontroller board

4. LPC2368 microcontroller board

5. External memory board (AL440B)

From the architecture planned, the 3-phase AC lines connect to ADE7758 and to HCPL-788J for being measured of their voltages and frequencies by the ADE7758 board which is interfaced with the LPC2368 microcontroller board in order to acquire the voltage and frequency values and also to initialize the ADE7758 for detecting fault signal at one time. Then the HCPL-788Jattenuates the voltage inputs and isolates the output signals. After that, the output signal is sent to become the signal inputs to the ADUC7024 microcontroller board for sampling waveforms and writing the sampling signals to the external memory board. In the ordinary state, the ADUC7024 microcontroller board will do the sampling and will write 
the data frames continuously, and in the same time, the LPC2368 microcontroller board will attain the voltage and frequency data from ADE7758, as well.

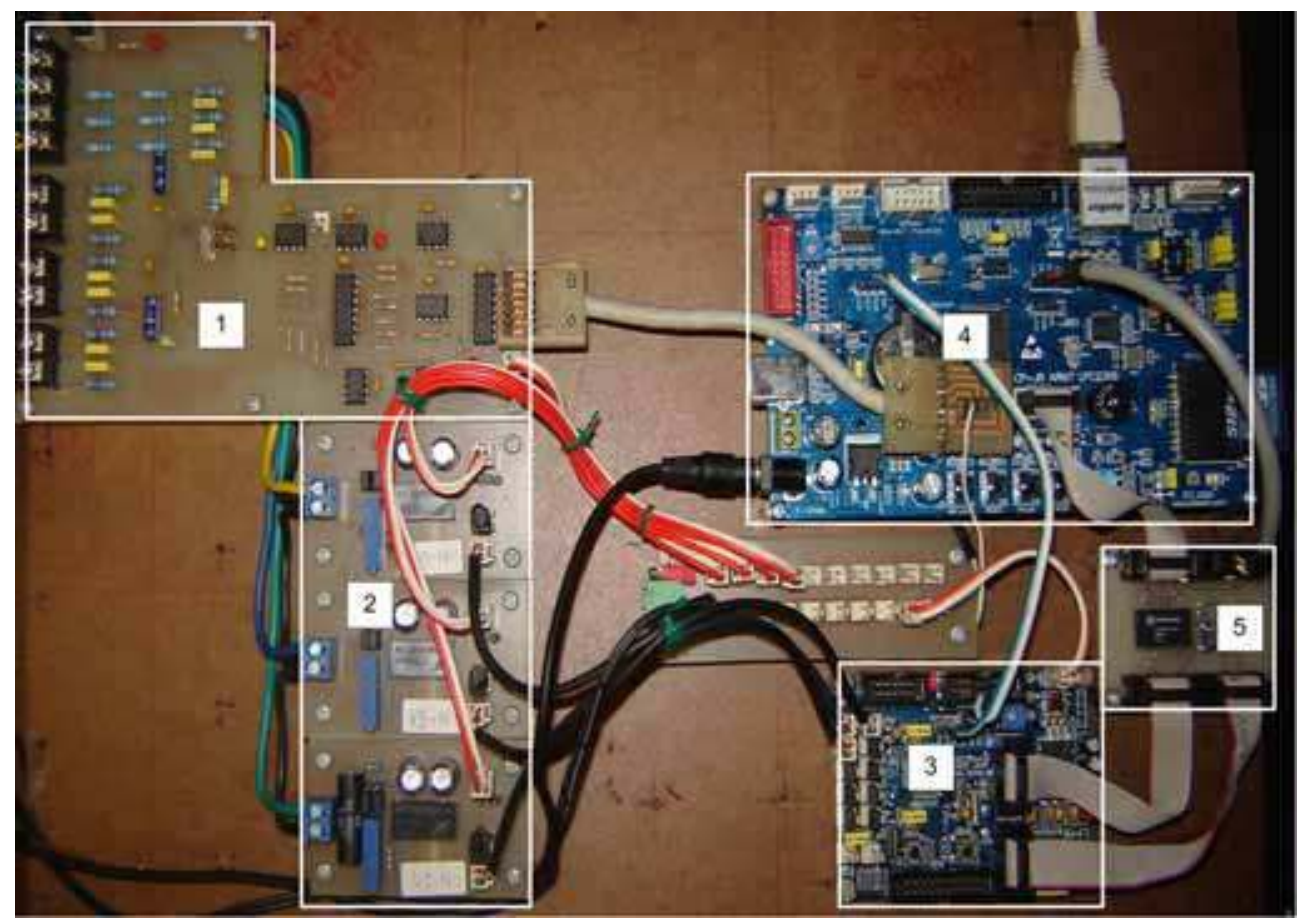

Fig. 7. The power quality monitoring system hardware

If the fault signal which is detected, in case, is an uncommon state, LPC2368 microcontroller board will control the ADUC7024 board to stop sampling and writing process temporarily, will read the sampling signal from the external memory board and will store the signal into SD-CARD. When it comes to the normal condition, both the microcontroller board will go back to their usual tasks as before.

In case of the network that has the connection with Transmission Control Protocol/ Internet Protocol (TCP/ IP) and User Datagram Protocol (UDP) is established between the LPC2368 and the PC or laptop, we can monitor the status of power quality monitoring system hardware via the network in real time. In addition, the fault data can be downloaded from the SD-CARD and displayed later when it is necessarily needed.

\section{Embedded software design}

The embedded software design is a flowchart of the two selected microcontrollers: ADUC7024 and LPC2368 used in this chapter. It is to explain the process of the power quality monitoring system based on the embedded system. For this embedded software design, there are the sampling concept, the power quality monitoring concept, data frame and the Ethernet packet structures. 


\subsection{Sampling concept}

The flowchart of the sampling concept shown below is to illustrate how the concept direction works according to the objectives of this chapter.

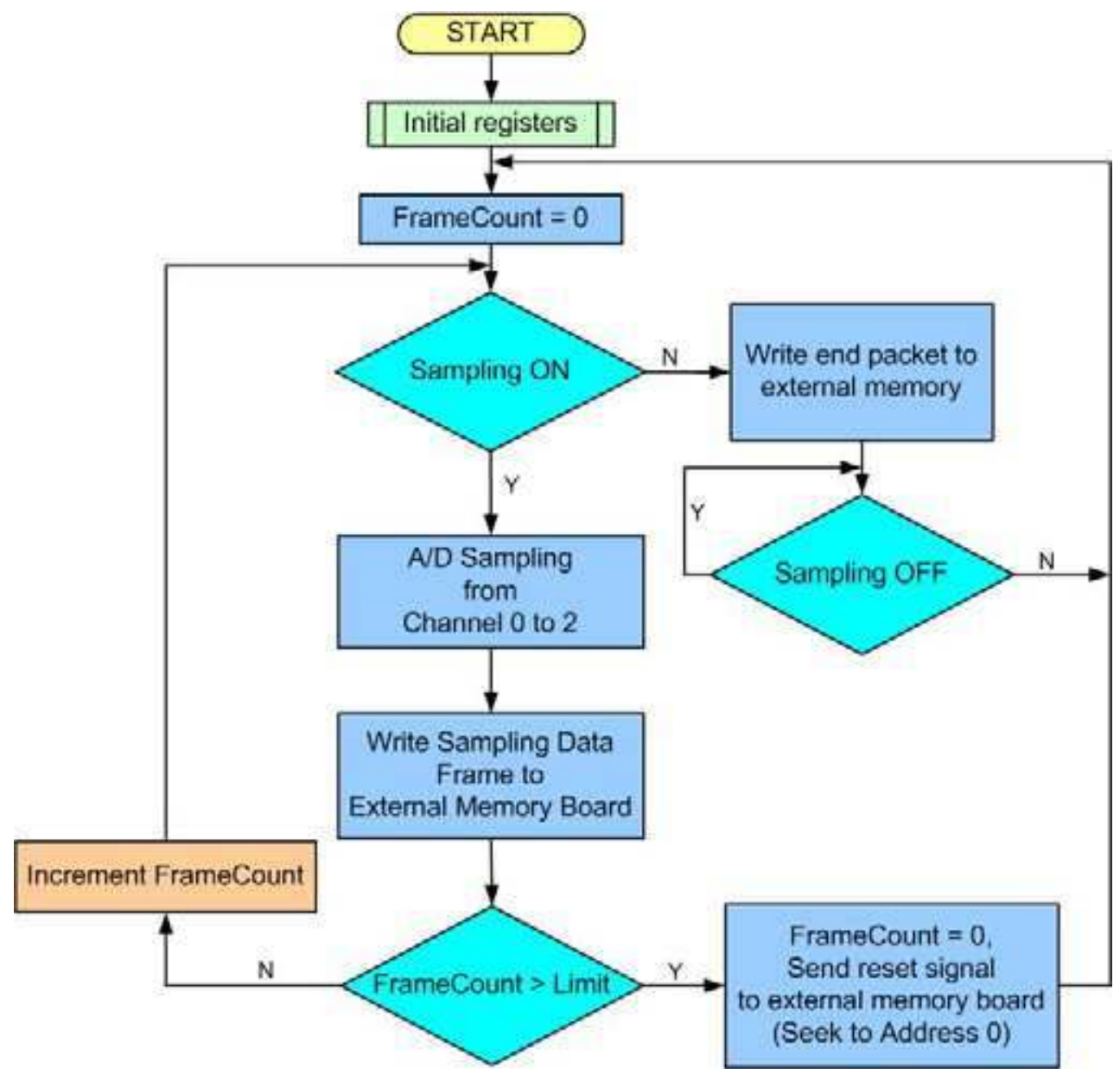

Fig. 8. Flow chart of the sampling concept

To explain the process of the sampling concept flow chart in Fig. 8., it is supposed to follow as the picture shown. After the used registers and utilized variables have been initialized, the procedures will work according to the conditions. That is, if the sampling condition is on operation, the procedure will do the sampling from the analog signals to the digital ones of the 3 channels. And the next procedure is to write the digital sampling data which are packed in the form of data frames to the external memory board. But if the counted data packet from the frame count value is excessive over the maximum limit, it will seek to the beginning of the first address in the external memory. Meanwhile, the data packet which is 
counted will be cleared. And if the counted data packet does not reach to the limit value, it will return to the sampling condition for rechecking.

In case, the sampling condition is off-operation, it will change the content of the data frame, will write it to the external memory and will return to the sampling condition to be rechecked. The procedures with the two sampling conditions mentioned above are done repeatedly time after time.

\subsection{Power quality monitoring concept}

It is generally a conceptual method to monitor power quality used with the embedded system of LPC2368 microcontroller board as shown in the flow chart of Fig. 9.

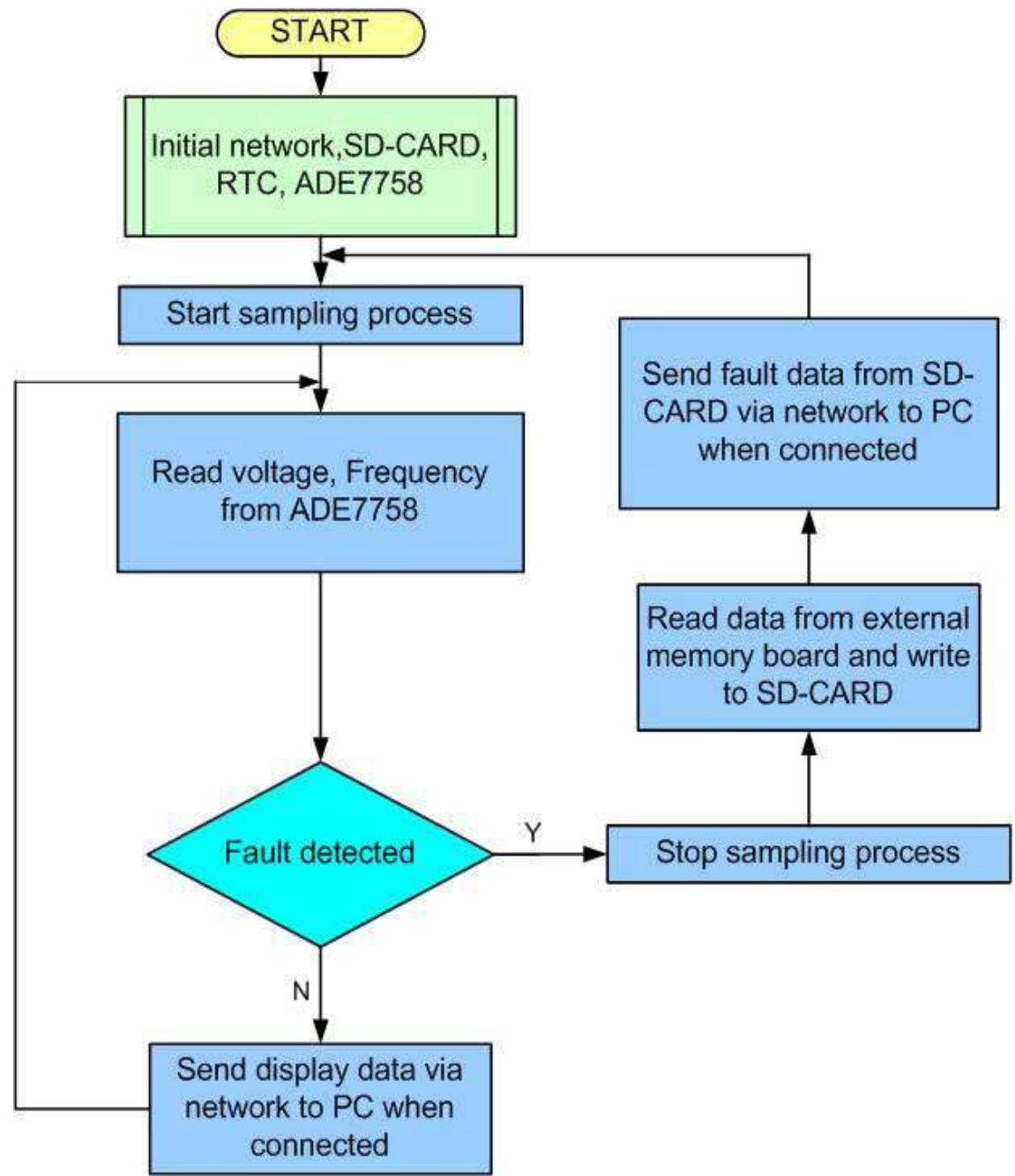

Fig. 9. Flow chart of the power quality monitoring concept 
To run the flowchart in order, it must initialize the network chip on LPC2368 microcontroller board, SD-CARD, real-time clock, the RTOS tasks that are modified from A Power Quality Monitoring System for Real-Time Detection of Power Fluctuations (Yingkayun \& Premrudeepreechacharn, 2008) and ADE7758 board to get ready for operating its functions. Next, LPC2368 microcontroller sends a control signal to ADUC7024 microcontroller board in order to start doing the sampling process. It reads the voltage and frequency data from ADE7758 board. In case, there is no any fault signals, which are read by LPC2368 microcontroller, the display information of the following values: the data of voltage; frequency; date; time; status and so on, will be sent via network to PC or laptop when it is connected. The LPC2368 microcontroller will take turn to operate its function repeatedly from the start once more. But if ADE7758 board detects the fault signals, it will send the fault signal to LPC2368 microcontroller which stops the sampling process, then, reads the fault data from the external memory board and writes the fault data in SD-CARD for storing. The fault data will be sent to PC or laptop via network when it is connected. LPC2368 microcontroller will start doing the process once more after receiving the next fault signals.

\subsection{Data frame}

The data frame, sending to the external memory board, is defined with the head byte, the samples of the 3-phase voltages with 12-bits A/ D resolution and the tail byte. The data frame content is shown in Fig. 10.

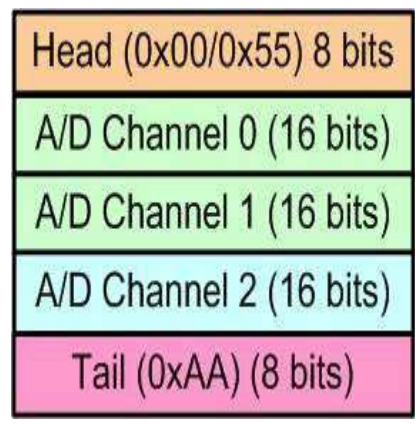

Fig. 10. Data frame

The example of data frame structure which is written in $\mathrm{C}$ structure format is shown below:

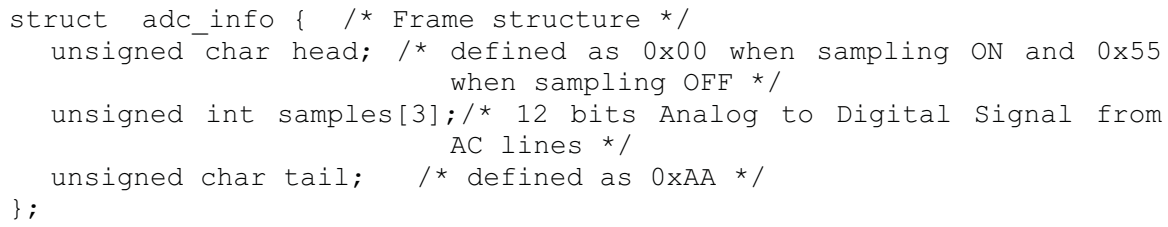

To calculate the floating point voltages from a raw A/ D sample, it can be expressed by 
Ethernet Frame

\begin{tabular}{|c|c|c|c|c|}
\hline $\begin{array}{c}\text { Ethernet } \\
\text { Header }\end{array}$ & $\begin{array}{c}\text { IP } \\
\text { Header }\end{array}$ & $\begin{array}{c}\text { UDP } \\
\text { Header }\end{array}$ & $\begin{array}{c}\text { Data } \\
\text { (Ethemet Stream Structure) }\end{array}$ & $\begin{array}{c}\text { Ethernet } \\
\text { Trailer }\end{array}$ \\
\hline
\end{tabular}

Fig. 11. UDP within Ethernet frame

The structures of the Ethernet stream can be illustrated in C programming language are as followings:

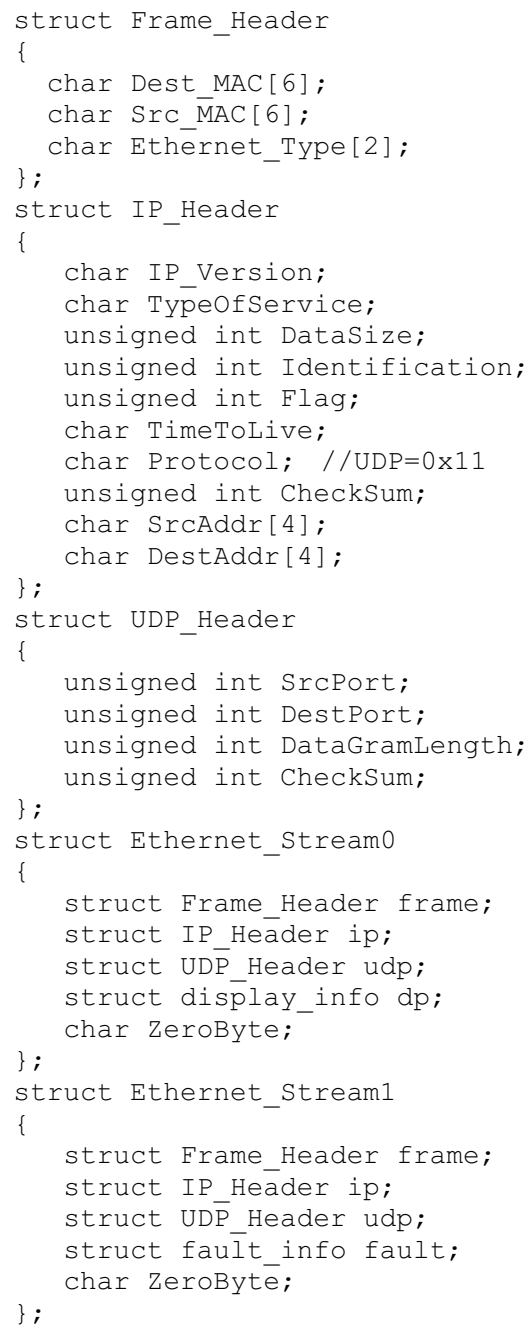

\section{Application software}

Application software is developed and written in Delphi 7 in order to control and to receive informational data from power quality monitoring system hardware and to save the data to 
the computer. Actually, this application gets the display information from the hardware mentioned above and then displays on the PC or laptop screen via the Ethernet network. The application can set date and time and can also receive the fault data from the referred hardware via the Ethernet network. The ordinary state and the uncommon one of the power lines are displayed by application software on PC or laptop shown in Fig. 12 and Fig. 13 respectively.

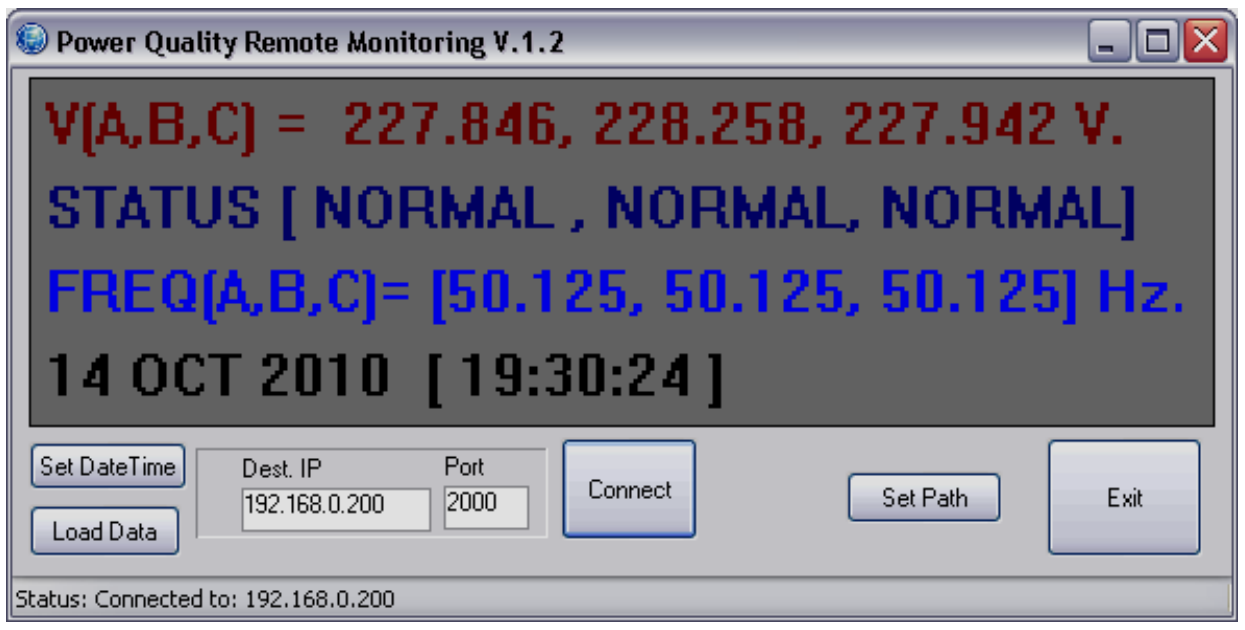

Fig. 12. Display window in ordinary state

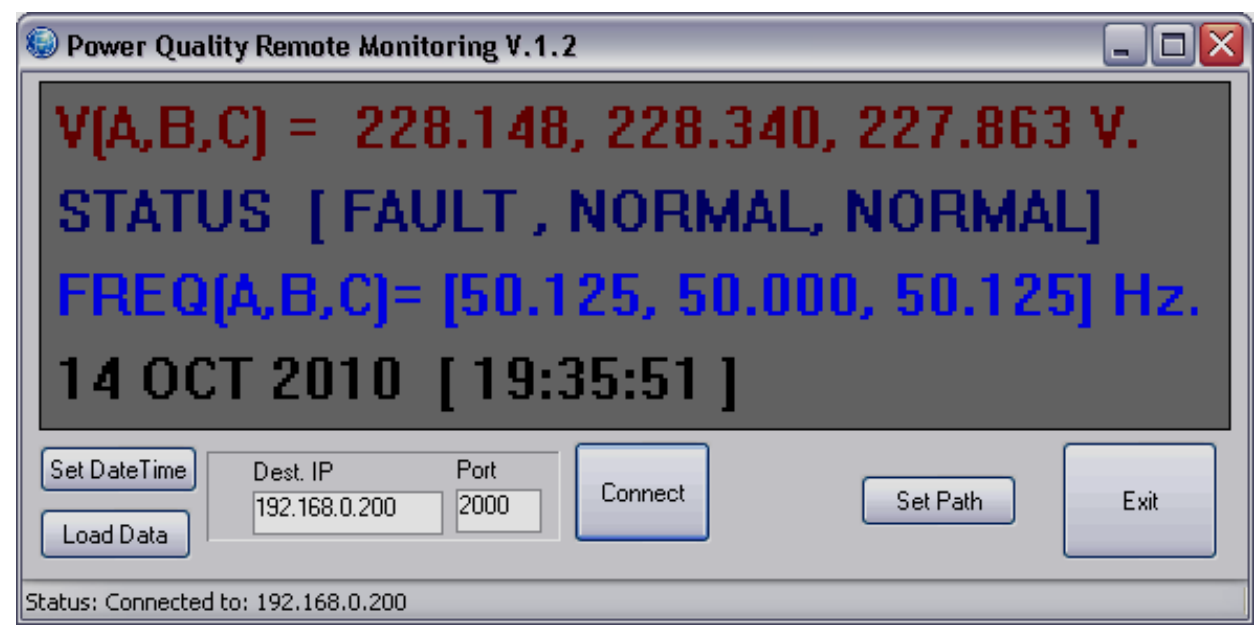

Fig. 13. Display window in uncommon state

And the researchers have developed the application software for displaying the fault signal which is sent from the monitoring hardware to PC or laptop in order to illustrate the fault data. This developed application software can either save in picture file or print out to take 
the data under the considerable analysis of the cause in the faults at later time. The developed application software is shown in Fig. 14.

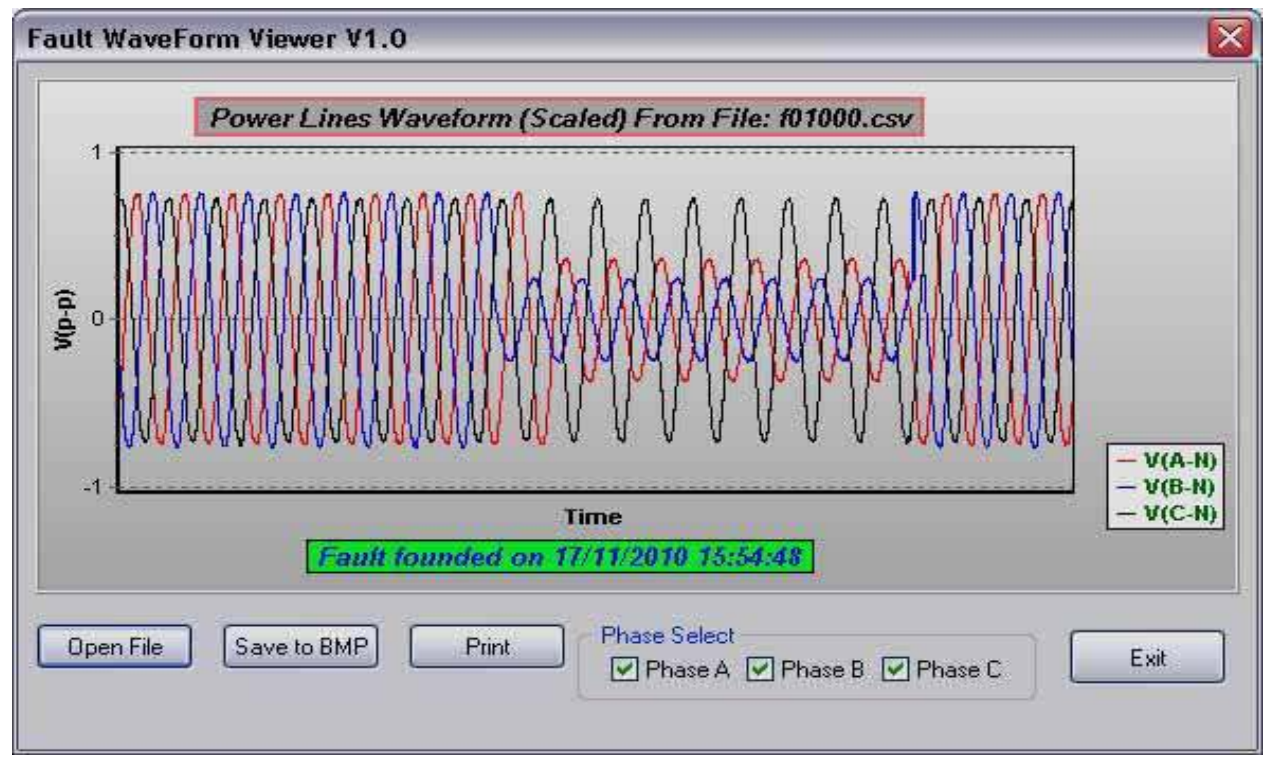

Fig. 14. Application software for waveform display

\section{Experimental results}

In order to communicate to the power quality monitoring hardware, application software is created to display and to plot the waveform. The application software connects to the monitoring hardware via the Ethernet network. It both gets the display information and saves the fault data to PC or laptop. It also shows the fault waveforms on the PC or laptop screen. The communication procedure connecting between a monitoring hardware to PC or laptop is to get the necessary information which is executed in the following steps:

1. A user sends a request signal with the application program to the power quality monitoring hardware.

2. The power quality monitoring hardware receives the request signal and establishes the connection to PC or laptop.

3. The power quality monitoring hardware sends the information to PC or laptop for displaying.

4. If any fault occurs, the fault data will be saved into the PC or laptop storage in the specific path.

5. A user can take the application software to display the fault waveform for investigating the problem in power lines from the saving path above.

When the connection procedures have been already established between the hardware and the computer in consequence, the user can monitor the voltage, the frequency and the fault in power lines on the PC or laptop screen from the remote site. Additionally, the user can also open the data file which is saved in the computer. 


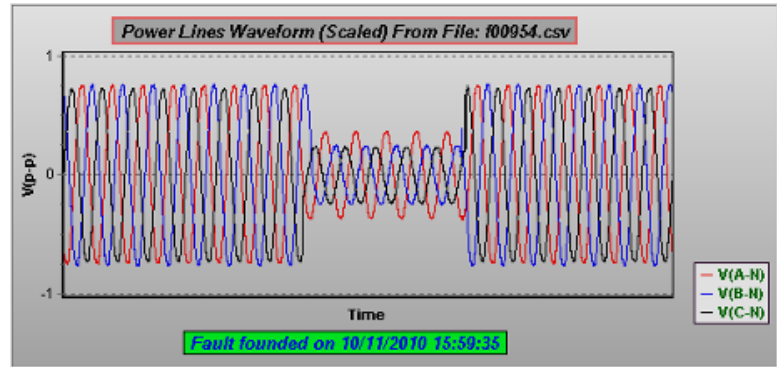

(a)

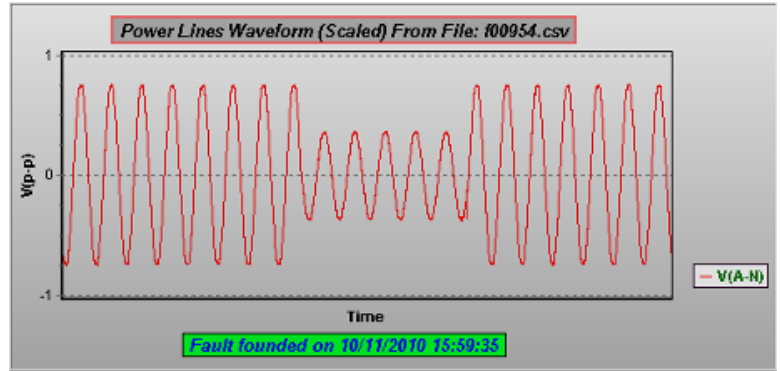

(b)

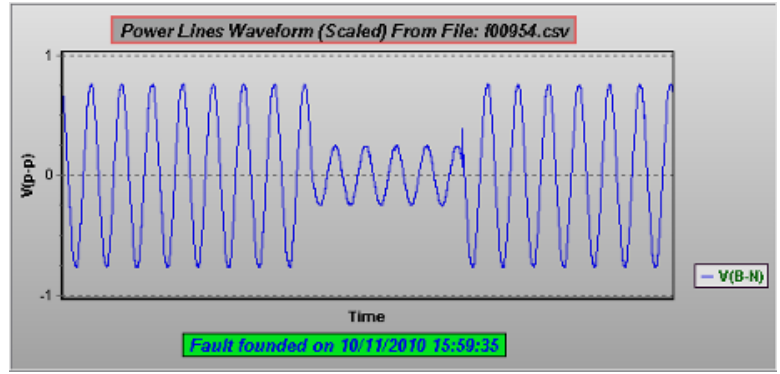

(c)

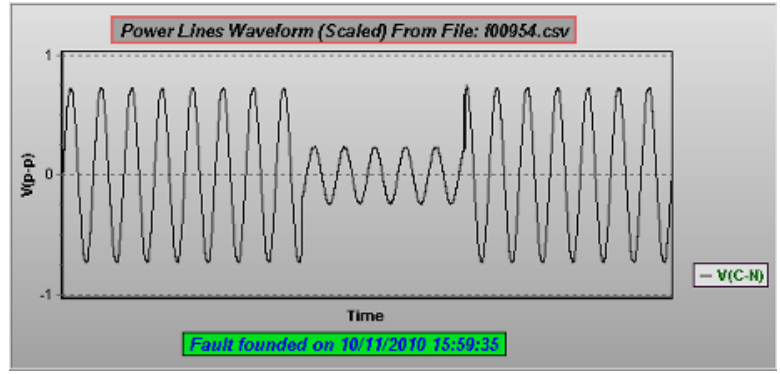

(d)

Fig. 15. (a) Voltage sags on phase A,B and C, (b) Voltage sag on phase A, (c) voltage sag on phase $B$ and $(d)$ voltage sag on phase $C$

For more advantage, the communication of the power quality monitoring hardware and PC or laptop is not only limited with the only one hardware but also connected to other hard- 
wares by executing more developed application programs that are shown in Fig. 12 and Fig. 13. The examples of the experimental result, that the data file is saved in the form of bitmap file (.BMP format) by the application program of Fig. 14, is illustrated in Fig. 15, Fig. 16 and Fig. 17.

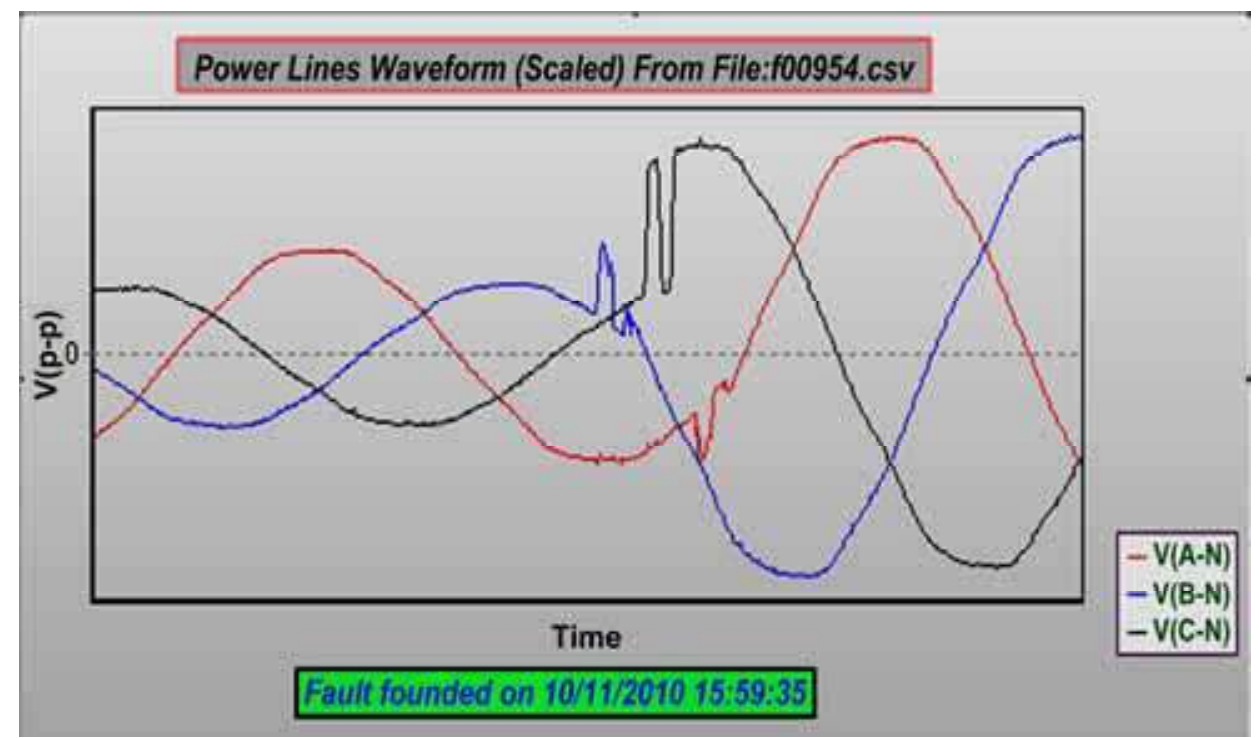

Fig. 16. Zoomed signals of voltage sags from Fig.15(a)

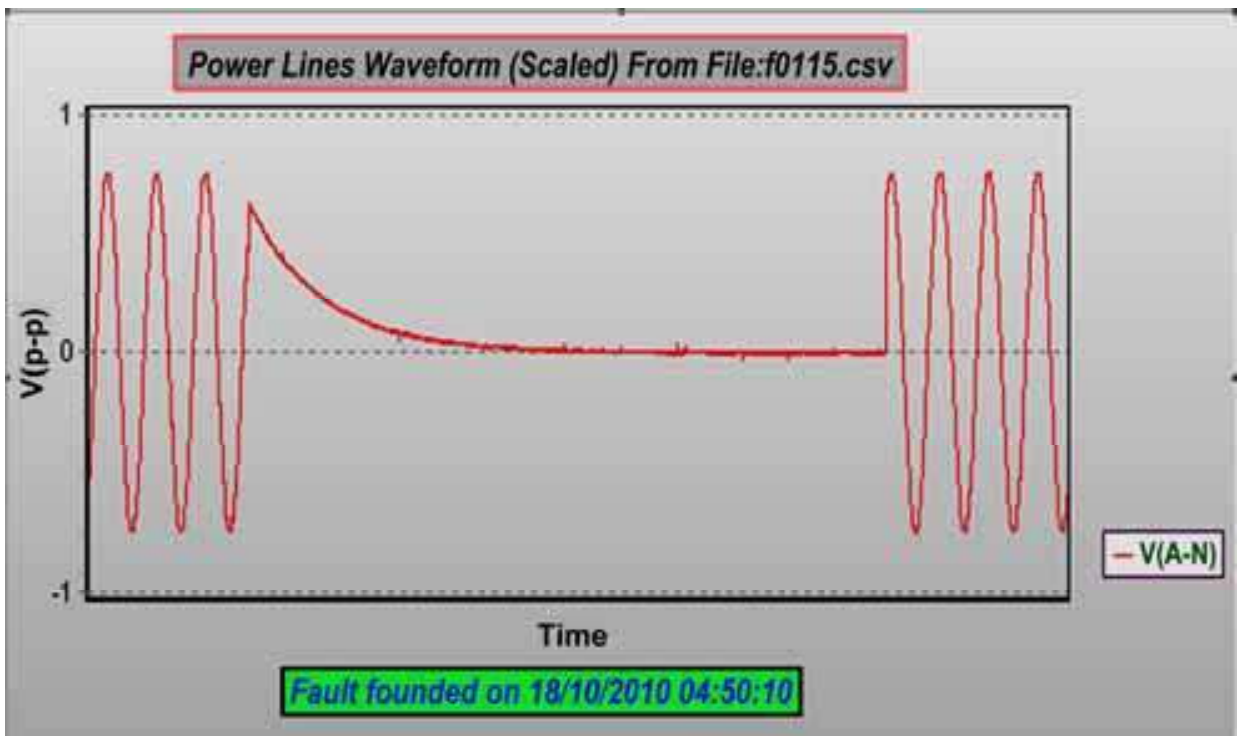

Fig. 17. Interruption on phase A (measured on a single phase system) 
Fig. 15(a) is the picture of sags for all 3 phases. The experimental results appeared in Fig. 15(b), (c) and (d) are the examples of sag in phase A, B and C chronically from Fig. 15(a). It is to separate the signal for testing each one in each phase that is easily studying.

Fig.16 is the zoomed picture from Fig. 15(a) to show the detail characteristics of the fault signals in each phase.

Another experiment of this chapter is applied to detect the fault on a single phase system. From Fig. 17 shown above is an example of the interruption for a short time.

\section{Conclusion and future work}

A power quality monitoring system via the Ethernet network based on the embedded system has been proposed in this chapter in order to monitor the power quality in case of faults detection and also to measure voltage and frequency in power lines. ADUC7024 and LPC2368 of ARM7 microcontroller are selected to apply in the power quality monitoring system for not only detecting the fault signals that cause any problems in either the system or the end user equipment but also reading and writing them in real time of power fluctuation. Moreover, the fault signal data can be sent and stored in SD-CARD to display later on the screen of PC or laptop at the site place. However, the users can download and analyze the fault signal data which have already sent and stored in SD-CARD via the Ethernet network using TCP/ IP and UPD protocol at some other time when of necessity needed.

For future work, the researchers tend to substitute ARM7 with ARM9 in order to monitor power quality and to detect the transient in power lines. In any case, the researchers have always concerned with the same primitive ideas and objectives.

\section{Acknowledgements}

The authors gratefully acknowledge to National Science and Technology Development Agency (NSTDA), Ministry of Science and Technology of Thailand, Thailand Research Fund (TRF), and Provincial Electricity Authority (PEA) for supports.

\section{References}

Auler, L.F. \& d'Amore, R. (2003). Power Quality Monitoring and Control using Ethernet Networks, Proceedings of 10th International Conference on Harmonics and Quality of Power, pp. 208-213, ISBN 0-7803-7671-4, Rio de Janeiro, Brazil, October 6-9, 2002

Auler, L.F. \& d' Amore, R. (2009). Power Quality Monitoring Controlled Through Low-Cost Modules, IEEE Transactions on Instrumentation and Measurement, Vol.58, No.3, (March 2009), pp. 557-562, ISSN 0018-9456

Baggini, A. B. (2008). Handbook of POWER QUALITY, WILEY, ISBN 978-0-470-06561-7, Wiltshire, Great Britain

Batista, J.; Alfonso, JL. \& Martins, J.S. (2004). Low-Cost Power Quality Monitor based on a PC, Proceeding of ISIE'03 IEEE International Symposium on Industrial Electronics, pp. 323-328, ISBN 0-7803-7912-8, Rio de Janeiro, Brazil, June 9-11, 2003

Dugan, R.C.; McGranaha, M.F.; Santoso, S. \& Beaty, H. W. (2002). Electrical Power Systems Quality, McGraw-Hill, ISBN 0-07-138622-X, New York, USA 
Hong, D.; Lee J. \& Choi, J. (2006). Power Quality Monitoring System using Power Line Communication, Proceeding of ICICS 2005 Fifth International Conference on Information, Communications and Signal Processing, pp. 931-935, ISBN 0-7803-9283-3, Bangkok, Thailand, December 6-9, 2005

Rahim bin Abdullah, A. \& Zuri bin Sha'ameri, A. (2005). Real-Time Power Quality Monitoring System Based on TMS320CV5416 DSP Processor, Proceeding of PEDS 2005 International Conference on Power Electronics and Drives Systems, pp. 16681672, ISBN 0-7803-9296-5, Kuala Lumpur, Malaysia, November 28 - December 1, 2005

Salem, M.E.; Mohamed, A.; Samad, S.A. \& Mohamed, R. (2006). Development of a DSPBased Power Quality Monitoring Instrument for Real-Time Detection of Power Disturbances, Proceedings of PEDS 2005 International Conference on Power Electronics and Drives Systems, pp. 304-307, ISBN 0-7803-9296-5, Kuala Lumpur, Malaysia, November 28 - December 1, 2005

So A.; Tse, N.; Chan W.L. \& Lai, L.L. (2000). A Low-Cost Power Quality Meter for Utility and Consumer Assessments, Proceeding of IEEE International Conference on Electric Utility Deregulation and Restructuring and Power Technologies, pp. 96-100, ISBN 07803-5902-X, City University London, UK, April 4-7, 2000

Yang, G.H. \& Wen, B.Y. (2006). A Device for Power Quality Monitoring Based on ARM and DSP, Proceedings of IEIEA 2006 The 1st IEEE Conference on Industrial Electronics and Applications, pp. 1-5, ISBN 0-7803-9513-1, Marina Mandarin Hotel, Singapore, May 24-26, 2006

Yingkayun, K. \& Premrudeepreechacharn S. (2009). A Power Quality Monitoring System for Real-Time Detection of Power Fluctuations, Proceeding of NAPS'08 The 40 ${ }^{\text {th }}$ North American Power Symposium, pp. 1-5, ISBN 978-1-4244-4283-6, Calgary, Canada, September 28-30, 2008

Yingkayun, K.; Premrudeepreechacharn S. \& Oranpiroj, K. (2009). A Power Quality Monitoring for Real-Time Fault Detection, Proceedings of ISIE 2009 IEEE International Symposium on Industrial Electronics, pp. 1846-1851, ISBN 978-1-42444347-5, Seoul, Korea, July 5-8, 2009 


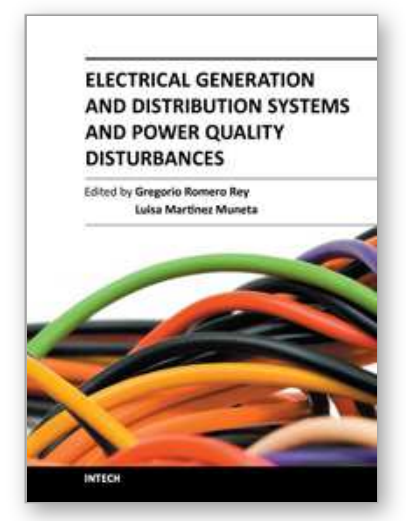

\section{Electrical Generation and Distribution Systems and Power Quality Disturbances}

Edited by Prof. Gregorio Romero

ISBN 978-953-307-329-3

Hard cover, 304 pages

Publisher InTech

Published online 21, November, 2011

Published in print edition November, 2011

The utilization of renewable energy sources such as wind energy, or solar energy, among others, is currently of greater interest. Nevertheless, since their availability is arbitrary and unstable this can lead to frequency variation, to grid instability and to a total or partial loss of load power supply, being not appropriate sources to be directly connected to the main utility grid. Additionally, the presence of a static converter as output interface of the generating plants introduces voltage and current harmonics into the electrical system that negatively affect system power quality. By integrating distributed power generation systems closed to the loads in the electric grid, we can eliminate the need to transfer energy over long distances through the electric grid. In this book the reader will be introduced to different power generation and distribution systems with an analysis of some types of existing disturbances and a study of different industrial applications such as battery charges.

\section{How to reference}

In order to correctly reference this scholarly work, feel free to copy and paste the following:

Krisda Yingkayun and Suttichai Premrudeepreechacharn (2011). A Power Quality Monitoring System Via the Ethernet Network Based on the Embedded System, Electrical Generation and Distribution Systems and Power Quality Disturbances, Prof. Gregorio Romero (Ed.), ISBN: 978-953-307-329-3, InTech, Available from: http://www.intechopen.com/books/electrical-generation-and-distribution-systems-and-power-qualitydisturbances/a-power-quality-monitoring-system-via-the-ethernet-network-based-on-the-embedded-system

\section{INTECH}

open science | open minds

\section{InTech Europe}

University Campus STeP Ri

Slavka Krautzeka 83/A

51000 Rijeka, Croatia

Phone: +385 (51) 770447

Fax: +385 (51) 686166

www.intechopen.com

\section{InTech China}

Unit 405, Office Block, Hotel Equatorial Shanghai

No.65, Yan An Road (West), Shanghai, 200040, China

中国上海市延安西路65号上海国际贵都大饭店办公楼 405 单元

Phone: +86-21-62489820

Fax: $+86-21-62489821$ 
(C) 2011 The Author(s). Licensee IntechOpen. This is an open access article distributed under the terms of the Creative Commons Attribution 3.0 License, which permits unrestricted use, distribution, and reproduction in any medium, provided the original work is properly cited. 